\title{
ANÁLISIS DE PLANIFICACIÓN DE TRAYECTORIAS LIBRES DE COLISIONES CON EL OPERADOR HUMANO EN SISTEMAS ROBÓTICOS DE TIPO SUPERNUMERARIO
}

\author{
D. Martínez-Pascual ${ }^{1}$, J. Álvarez-Pastor ${ }^{1}$, F.J. Verdú-García ${ }^{1}$, M. Arnau-Papí ${ }^{1}$, L.D. Lledó1 ${ }^{1}$ F. Lopez-Labrador ${ }^{2}$ \\ M.Á. Bernal ${ }^{2}$ y N. García-Aracil ${ }^{1}$ \\ ${ }^{1}$ Universidad Miguel Hernández, Av. de la Universidad, s/n, 03202, Elche. david.martinezp@umh.es \\ ${ }^{2}$ Eiffage Energia, Ctra. Mahora Km 3,2 02006 Albacete
}

\section{Resumen}

Con objeto de disminuir los trastornos musculoesqueléticos en el ámbito laboral, se plantea la posibilidad de emplear dispositivos robóticos de tipo supernumerario que permitan apoyar diversos tipos de tareas durante la realización de trabajos de mantenimiento. Además, se pretende que este tipo de dispositivos sea capaz de actuar de forma cooperativa con el usuario de forma autónoma o semi-autónoma. Para ello, se precisa desarrollar un sistema capaz de evitar la colisión con el usuario. En este trabajo se analiza el uso de planificadores de trayectorias para la evasión de los brazos humanos durante tareas de mantenimiento mediante un modelo simulado.

Palabras clave: Robot supernumerario, planificadores de trayectorias, Robotics Operating System (ROS)

\section{INTRODUCCIÓN}

Los trastornos musculoesqueléticos (TME) son alteraciones que abarcan desde incomodidad, molestias o dolores hasta cuadros médicos más graves que obligan a solicitar la baja laboral e incluso a recibir tratamiento médico. Pueden afectar a los músculos, articulaciones, tendones, ligamentos, huesos y nervios del cuerpo [1].

Las demandas físicas de las tareas realizadas son de los factores de riesgo más asociados a los TME, particularmente las posturas forzadas, la manipulación de cargas, la aplicación de fuerzas y la repetitividad de las acciones. Analizando los resultados de la VII Encuesta Nacional de Condiciones de Trabajo relacionados con los trastornos musculoesqueléticos, los movimientos repetitivos de manos o brazos eran los que afectaban en mayor medida a los encuestados (59\%), seguidos por la adopción de posturas dolorosas o fatigantes $(35,8 \%)$ y la manipulación de cargas $(23,3 \%)[4]$.

Hacer frente a los TME y los riesgos psicosociales que conllevan exige la adopción de medidas en el puesto de trabajo. Estas medidas se pueden dividir en dos grandes líneas de actuación: 1) adecuación del puesto de trabajo y 2) desarrollo de ayudas técnicas avanzadas. Dentro de las ayudas técnicas avanzadas, surge el proyecto SecondArmS. Este proyecto tiene como objetivo el desarrollo de dispositivos robóticos bimanuales que ayuden al usuario en tareas de manipulación de cargas y en la realización de movimientos repetitivos. Además, el dispositivo desarrollado permitirá asistir al usuario en la adopción de posturas fatigantes o dolorosas durante la realización de trabajos de mantenimiento, como por ejemplo cuando se realizan tareas de mantenimiento por encima del nivel de la cabeza.

En cuanto al control del sistema, los brazos podrán ser teleoperados, pero también se propone desarrollar un sistema que permita mover los manipuladores robóticos de forma autónoma o semi-autónoma, capaz de evitar la colisión con el usuario. Por tanto, el problema pasa por generar trayectorias entre una configuración inicial y final para los manipuladores en el espacio libre, i.e., en el espacio no ocupado por el usuario o por el propio dispositivo robótico.

MoveIt! es un software ampliamente utilizado para manipulación en ROS [3], el cual permite escoger entre diferentes librerías de planificación de trayectorias. Entre ellas se encuentra la librería Open Motion Planning Library (OMPL) [7], y es la librería de planificación de trayectorias configurada por defecto en MoveIt!.

La librería OMPL incluye planificadores basados en muestreos aleatorios. Este concepto de planificadores se basa en un muestreo del espacio de estados del robot para así proporcionar una respuesta rápida al problema de planificación. Dentro de este tipo de planificadores, podríamos distinguir dos subtipos:

- Planificadores single-query. Buscan respuesta a una única planificación entre configuración inicial y final. Estos planificadores se basan en la construcción de árboles de estados conectados por movimientos válidos. Dentro de este 
conjunto se encuentran los planificadores RRT, EST, KPIECE, STRIDE, PDST o FMT, y sus variantes (TRRT, BiTRRT, RRTConnect, RRT*, SBL, BiEST, ProjEST, BKPIECE, LBKPIECE).

- Planificadores multi-query. Este tipo de planificadores construye un mapa de caminos válidos que cubre todo el espacio de estados. Una vez construido el mapa, éste servirá para realizar múltiples peticiones de planificación entre configuración inicial y final. En esta categoría se encuentran los planificadores PRM y SPARS, así como sus variantes (PRM*, LazyPRM, LazyPRM*, SPARS2).

Para desarrollar un sistema que evite colisionar con el usuario, se considerará la posibilidad de emplear los planificadores de trayectorias de la librería OMPL. Cabe destacar que, debido a la naturaleza de la aplicación presentada, se propone investigar el rendimiento de los planificadores como single-query, incluidos los planificadores multi-query PRM, SPARS y sus variantes.

A lo largo de las siguientes secciones se presenta un modelo simulado del sistema SecondArmS y la evaluación del rendimiento de los planificadores en base a diferentes métricas.

\section{MATERIALES Y MÉTODOS}

\subsection{DESCRIPCIÓN DEL SISTEMA}

Para analizar el comportamiento en simulación, se ha desarrollado un modelo del sistema SecondArmS para la plataforma Robotics Operating System (ROS) [6]. El modelo se implementa mediante ficheros URDF (Unified Robotic Description Format). Los ficheros URDF son ficheros en formato XML que permiten describir los elementos de un robot y las relaciones entre ellos en ROS.

El sistema propuesto estará formado por tres componentes principales: los brazos manipuladores robóticos, una plataforma elevadora y el operador humano. Todos estos elementos se modelan en un único fichero URDF, de modo que se tendrá en todo instante información de todos los elementos del sistema y la interacción entre ellos. La Figura 1 muestra una representación del sistema implementado.

Debido a que el sistema se encuentra en desarrollo, el modelo implementado no se trata de un modelo definitivo. No obstante, el modelo se desarrolla con las dimensiones esperadas del sistema final. En las siguientes secciones se

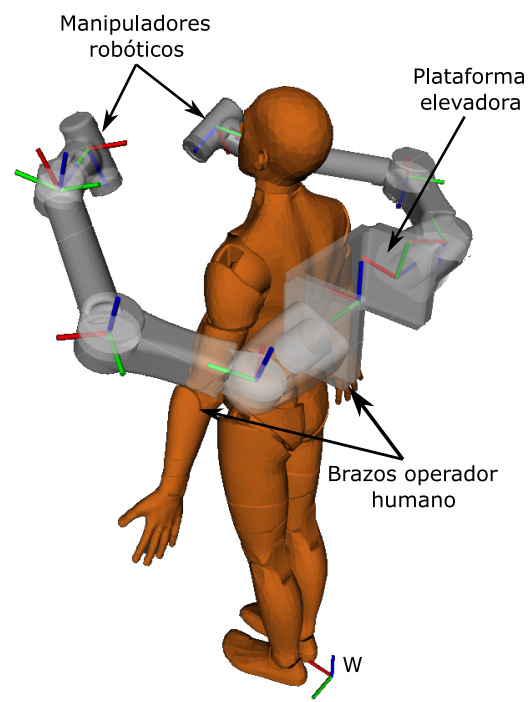

Figura 1: Modelo del sistema SecondArmS implementado en ROS $(\mathrm{W}=$ Sistema de referencia del mundo)

presenta de forma más detallada cada uno de los elementos principales que componen el conjunto del modelo.

\subsubsection{Brazos manipuladores}

El sistema posee dos manipuladores robóticos colaborativos con muñeca no esférica de 6 grados de libertad (GDL), lo que permite alcanzar los puntos del espacio de trabajo con diferentes orientaciones (3 grados de libertad para posición y 3 grados de libertad para orientación). Cada grado de libertad se modela como una articulación rotacional en el modelo URDF.

Cabe destacar que los manipuladores robóticos se diseñan como una extensión de los brazos humanos, por lo que el alcance de los brazos robóticos será similar al alcance de un brazo humano. Esto puede observarse en la Figura 2, donde se hace una representación del espacio de trabajo de la muñeca y alcanzable de los manipuladores robóticos con respecto al operador humano. Dichos espacios de trabajo han sido representados mediante métodos Montecarlo, resolviendo la cinemática directa de los manipuladores para un número $N$ de valores articulares aleatorios dentro del rango articular.

\subsubsection{Plataforma elevadora}

El sistema SecondArmS está planteado de tal forma que el usuario porte una mochila con una plataforma que eleve o baje la base de los brazos robóticos. Esto conllevará un aumento del espacio de trabajo, y además permitirá al usuario 


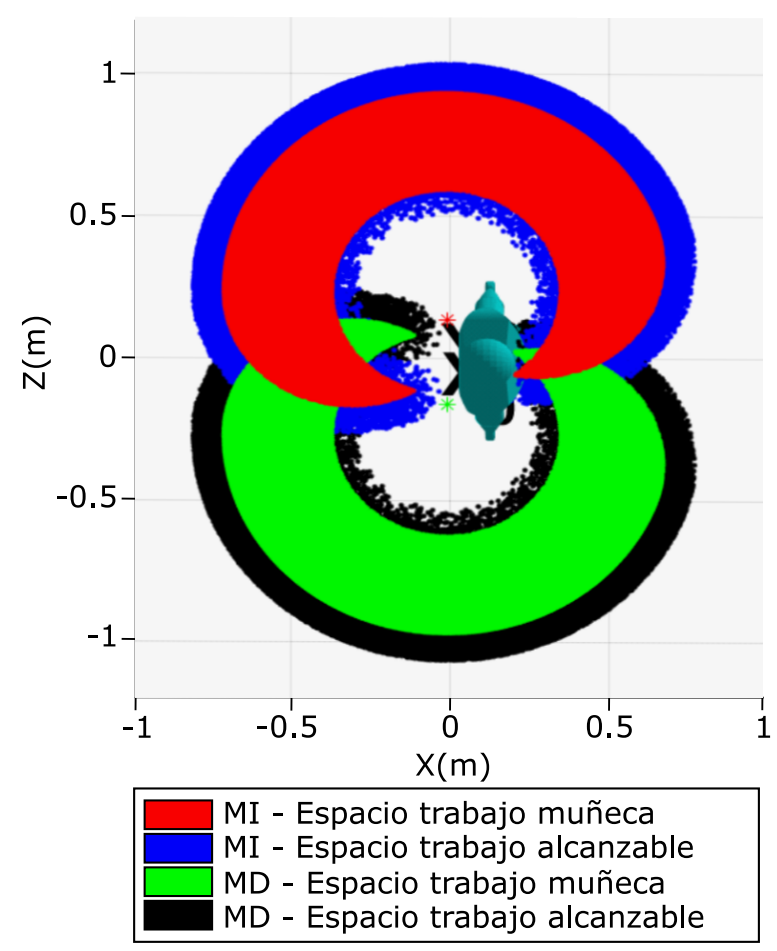

Figura 2: Representación del espacio de trabajo de la muñeca y espacio de trabajo alcanzable en el plano XZ de los manipuladores robóticos izquierdo (MI) y derecho (MD).

acomodarse el sistema según la tarea a realizar.

Dicha plataforma se desplazará sobre la mochila mediante un husillo con una carrera de $450 \mathrm{~mm}$, desde la cadera hasta los hombros del operario. Dicho desplazamiento se modelará como una articulación prismática, sobre la cual se fijan las bases de los brazos manipuladores.

\subsubsection{Operador humano}

El propósito del proyecto SecondArmS es desarrollar un sistema de brazos robóticos colaborativos con el usuario. Por ello, se implementará el operador humano como parte del sistema. Esto permitirá tener en cuenta al usuario, de forma que se podrá evitar colisiones con el mismo.

El operador humano se modelará con la cabeza, torso y miembros inferiores estáticos. No obstante, se modelará como partes móviles las articulaciones de los miembros superiores. Se modelan los movimientos de abducción/aducción de hombro, flexión/extensión de hombro, rotación interna/externa de codo y flexión/extensión de codo, siendo para cada articulación el rango de movimiento definido en [5].

El propósito de modelar el movimiento de los brazos del usuario se debe a que se desea estudiar el comportamiento del sistema simulado de forma que sea lo más fiel posible a la realidad, probando diferentes configuraciones del usuario y estudiando el desempeño del sistema completo. En un futuro se pretende desarrollar una aplicación que permita conocer la posición del brazo mediante unidades magnético inerciales (IMU), basado en trabajos previos del grupo de investigación [2], para así lograr que los brazos robóticos eviten colisionar con el usuario.

\subsection{EVALUACIÓN DE LOS PLANIFICADORES}

Para valorar el rendimiento de los planificadores incluidos en OMPL se establece una pose inicial y final del brazo robótico, para las que se debe planificar un camino libre de obstáculos. Como se desea evaluar el comportamiento del sistema de forma fiel a la realidad, se articula el brazo del usuario, generando así un obstáculo entre las configuraciones inicial y final del manipulador. Además, para una correcta evaluación del rendimiento de los planificadores, se planificará $n$ veces un camino entre la configuración inicial y final.

El rendimiento de los planificadores se evaluará en base a 3 medidas para una misma configuración inicial y final: número de intentos de planificación con éxito, tiempo de planificación y longitud del camino generado.

Los intentos de planificación se analizan como el número de trayectorias planificadas con éxito con respecto al número total de intentos de planificación. Para cada planificador, a mayor número de trayectorias planificadas con éxito se valora mejor desempeño. Por otra parte, el tiempo de planificación se mide como el tiempo necesario para encontrar una trayectoria válida. Para cada planificador, a menor tiempo de planificación se califica como mejor rendimiento. Por último, se evalúa la longitud de las trayectorias planificadas. A menor longitud de trayectoria, mejor rendimiento. Asimismo, se considera como mejor rendimiento del planificador un menor rango intercuartílico para las longitudes de las trayectorias.

Cabe destacar que a partir de los planificadores OMPL se obtendrán las trayectorias para los brazos robóticos como referencias en el espacio articular. No obstante, puede aproximarse el cálculo de la longitud total del camino planificado haciendo uso de la resolución de la cinemática directa del manipulador. Si resolvemos la cinemática directa para cada referencia articular, obtendremos una matriz de transformación homogénea a partir de la 
XLII Jornadas de Automática

cual podemos obtener la posición en el espacio cartesiano. Esto se expresa en la Ecuación 1, donde $r_{i}^{E}$ representa las referencias generadas en el espacio cartesiano y $F K$ la resolución de la cinemática directa del manipulador, siendo $r_{i}^{Q}$ un vector con los valores articulares de los 6 GDL del brazo robótico.

$$
r_{i}^{E}=F K\left(r_{i}^{Q}\right)=\left(\begin{array}{cc}
R_{i} & T_{i} \\
0 & 1
\end{array}\right)
$$

Para calcular la longitud total del camino planificado, se hará uso de la posición del efector final $T_{i}$ para cada referencia. El cálculo de la longitud total de la trayectoria $D_{s}^{g}$ se expresa en la Ecuación 2, donde $n$ es el número total de referencias generadas.

$$
D_{s}^{g}=\sum_{i=1}^{n}\left\|\overrightarrow{T_{i-1} T_{i}}\right\|
$$

\section{RESULTADOS}

Para evaluar el comportamiento de los planificadores en diferentes situaciones, se ha simulado dos tipos de tareas con uno de los brazos robóticos: una tarea por encima del nivel de la cabeza, y una tarea de manipulación frontal (Figura 3). Las posiciones y orientaciones de las configuraciones inicial y final del manipulador, la altura de la plataforma y los ángulos del brazo del operador humano se recogen en la Tabla 1.

Para tener una fuente de datos fiable acerca del porcentaje de éxito, el tiempo de cálculo y la longitud de las trayectorias, cada algoritmo de planificación se ha ejecutado 100 veces para la mismas configuraciones inicial y final. Asimismo, se ha establecido como tiempo máximo asumible de planificación 5 segundos. Cabe destacar que se ha escogido un tiempo asumible de planificación alto debido debido a la naturaleza
Modelado, Simulación y Optimización

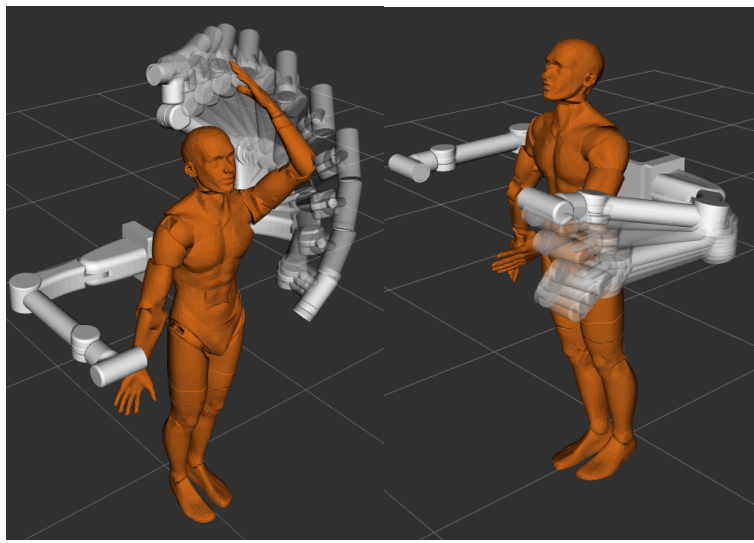

(a)

(b)

Figura 3: Simulaciones realizadas para evaluar el comportamiento de los planificadores de trayectorias durante tareas de mantenimiento (a) Simulación de tarea sobre la cabeza (b) Simulación de tarea frontal

de las tareas simuladas. En estos trabajos de mantenimiento el operador humano se supone estático, mientras que el manipulador robótico es el que realiza diferentes movimientos y tareas. Por ello, podemos suponer que los movimientos humanos son nulos y comprobar si tiempos de planificación altos logran un buen porcentaje de éxito y buenas trayectorias, especialmente los planificadores multi-query.

Los resultados obtenidos para la simulación de la tarea sobre la cabeza y para la tarea frontal se recogen en la Tabla 2. La Figura 4 y la Figura 5 muestran un gráfico boxplot de las longitudes de las trayectorias planificadas para ambas tareas.

Para la tarea por encima de la cabeza, todos los planificadores han sido capaces de encontrar un camino válido en el tiempo máximo establecido, a excepción de RRT (99\%), RRT* (94\%) y LazyPRM, incapaz de planificar un camino en el tiempo establecido. El planificador más rápido ha sido KPIECE, con un tiempo de planificación

Tabla 1: Parámetros de simulación para tarea sobre la cabeza (Tarea 1) y tarea frontal (Tarea 2). Las poses del efector final del manipulador robótico y la altura de la plataforma se referencia respecto al

\begin{tabular}{|c|c|c|c|}
\hline & & Tarea 1 & Tarea 2 \\
\hline \multirow{4}{*}{ Brazo operador humano } & Abducción hombro [rad] & 0.00 & 0.15 \\
\hline & Flexión hombro [rad] & 2.40 & 0.85 \\
\hline & Rotación interna codo [rad] & 0.55 & 0.60 \\
\hline & Extensión codo [rad] & 0.00 & 0.82 \\
\hline \multirow{2}{*}{ Pose de partida } & Posición $(\mathrm{x}, \mathrm{y}, \mathrm{z})[\mathrm{m}]$ & $(-0.12,0.08,1.91)$ & $(0.70,0.33,1.05)$ \\
\hline & Orientación (roll, pitch, yaw) [rad] & $(-1.47,0.00,-3.04)$ & $(-2.48,1.56,-2.48)$ \\
\hline \multirow{2}{*}{ Pose objetivo } & Posición $(\mathrm{x}, \mathrm{y}, \mathrm{z})[\mathrm{m}]$ & $(0.65,0.16,1.27)$ & $(0.70,0.33,1.40)$ \\
\hline & Orientación (roll, pitch, yaw) [rad] & $(2.75,-0.59,-1.06)$ & $(-2.6,1.35,-2.59)$ \\
\hline \multicolumn{2}{|c|}{ Altura plataforma elevadora $[\mathrm{m}]$} & 1.40 & 1.25 \\
\hline
\end{tabular}
sistema de coordenadas del mundo (W) 
Tabla 2: Porcentaje de éxito, tiempo de planificación (media y desviación estándar), mediana de longitud de trayectorias planificadas y rango intercuartílico de longitud de trayectorias (IQR)

\begin{tabular}{|c|c|c|c|c|c|c|c|c|}
\hline \multirow{2}{*}{ Planificador } & \multicolumn{4}{|c|}{ Tarea sobre la cabeza } & \multicolumn{4}{|c|}{ Tarea frontal } \\
\hline & $\begin{array}{c}\text { Porcentaje de } \\
\text { éxito }(\%)\end{array}$ & Tiempo (s) & Longitud (m) & IQR & $\begin{array}{c}\text { Porcentaje de } \\
\text { éxito }(\%)\end{array}$ & Tiempo (s) & Longitud (m) & IQR \\
\hline SBL & 100 & $0.0898 \pm 0.0166$ & 3.6546 & 0.9611 & 100 & $0.1102 \pm 0.0216$ & 2.3248 & 0.3852 \\
\hline EST & 100 & $0.0876 \pm 0.0281$ & 3.7863 & 1.6804 & 100 & $0.1173 \pm 0.0548$ & 2.4215 & 0.5884 \\
\hline LBKPIECE & 100 & $0.1061 \pm 0.0189$ & 3.2360 & 1.9616 & 100 & $0.1245 \pm 0.0266$ & 2.0960 & 0.1248 \\
\hline BKPIECE & 100 & $0.1455 \pm 0.0472$ & 3.3607 & 1.9193 & 100 & $0.1562 \pm 0.0545$ & 2.1493 & 0.2466 \\
\hline KPIECE & 100 & $0.0689 \pm 0.0165$ & 3.4994 & 1.8474 & 100 & $0.0990 \pm 0.0280$ & 2.2558 & 0.3063 \\
\hline RRT & 89 & $0.1350 \pm 0.3128$ & 3.4579 & 1.8704 & 89 & $0.1603 \pm 0.2114$ & 2.1479 & 0.2766 \\
\hline RRTConnect & 100 & $0.0877 \pm 0.0129$ & 3.2057 & 1.9582 & 100 & $0.1528 \pm 0.0320$ & 1.9342 & 0.0697 \\
\hline RRT $^{*}$ & 96 & $5.0442 \pm 0.0130$ & 3.2167 & 0.3823 & 96 & $5.0723 \pm 0.0203$ & 1.9760 & 0.0919 \\
\hline TRRT & 95 & $0.24335 \pm 0.5736$ & 3.3707 & 0.6079 & 95 & $0.2410 \pm 0.4876$ & 2.1072 & 0.2351 \\
\hline PRM & 100 & $5.0210 \pm 0.0115$ & 3.5343 & 1.3336 & 100 & $5.0385 \pm 0.0121$ & 2.3320 & 0.4922 \\
\hline $\mathrm{PRM}^{*}$ & 100 & $5.0296 \pm 0.0124$ & 3.6245 & 1.7851 & 100 & $5.0487 \pm 0.0165$ & 2.3346 & 0.4768 \\
\hline FMT & 100 & $1.2154 \pm 0.1174$ & 3.3362 & 0.4232 & 100 & $1.3544 \pm 0.0465$ & 2.1423 & 0.2770 \\
\hline PDST & 100 & $0.1160 \pm 0.0495$ & 3.7919 & 1.1903 & 100 & $0.1353 \pm 0.0613$ & 2.5961 & 0.7101 \\
\hline STRIDE & 100 & $0.0716 \pm 0.0237$ & 3.6188 & 1.8169 & 100 & $0.0989 \pm 0.0344$ & 2.1649 & 0.2674 \\
\hline BiTRRT & 100 & $0.0976 \pm 0.0239$ & 3.4454 & 1.8918 & 100 & $0.1228 \pm 0.0159$ & 2.1664 & 0.2251 \\
\hline BiEST & 100 & $0.0995 \pm 0.0210$ & 4.2499 & 1.5692 & 100 & $0.1146 \pm 0.0252$ & 2.6982 & 1.0340 \\
\hline ProjEST & 100 & $0.0822 \pm 0.0290$ & 3.6092 & 1.7365 & 100 & $0.1088 \pm 0.0396$ & 2.4773 & 0.4453 \\
\hline LazyPRM & 0 & $\mathrm{~N} / \mathrm{A}$ & $\mathrm{N} / \mathrm{A}$ & $\mathrm{N} / \mathrm{A}$ & 0 & $\mathrm{~N} / \mathrm{A}$ & $\mathrm{N} / \mathrm{A}$ & $\mathrm{N} / \mathrm{A}$ \\
\hline LazyPRM* & 100 & $5.0193 \pm 0.0155$ & 3.3457 & 1.9271 & 100 & $5.0469 \pm 0.01516$ & 2.0902 & 0.1910 \\
\hline SPARS & 100 & $5.0416 \pm 0.0163$ & 3.7740 & 1.7463 & 100 & $5.0606 \pm 0.0210$ & 2.4496 & 0.9427 \\
\hline SPARS2 & 100 & $5.0205 \pm 0.0141$ & 4.1170 & 1.5801 & 100 & $5.0349 \pm 0.0160$ & 2.8066 & 1.1204 \\
\hline
\end{tabular}

$\mathrm{N} / \mathrm{A}=$ No Aplicable

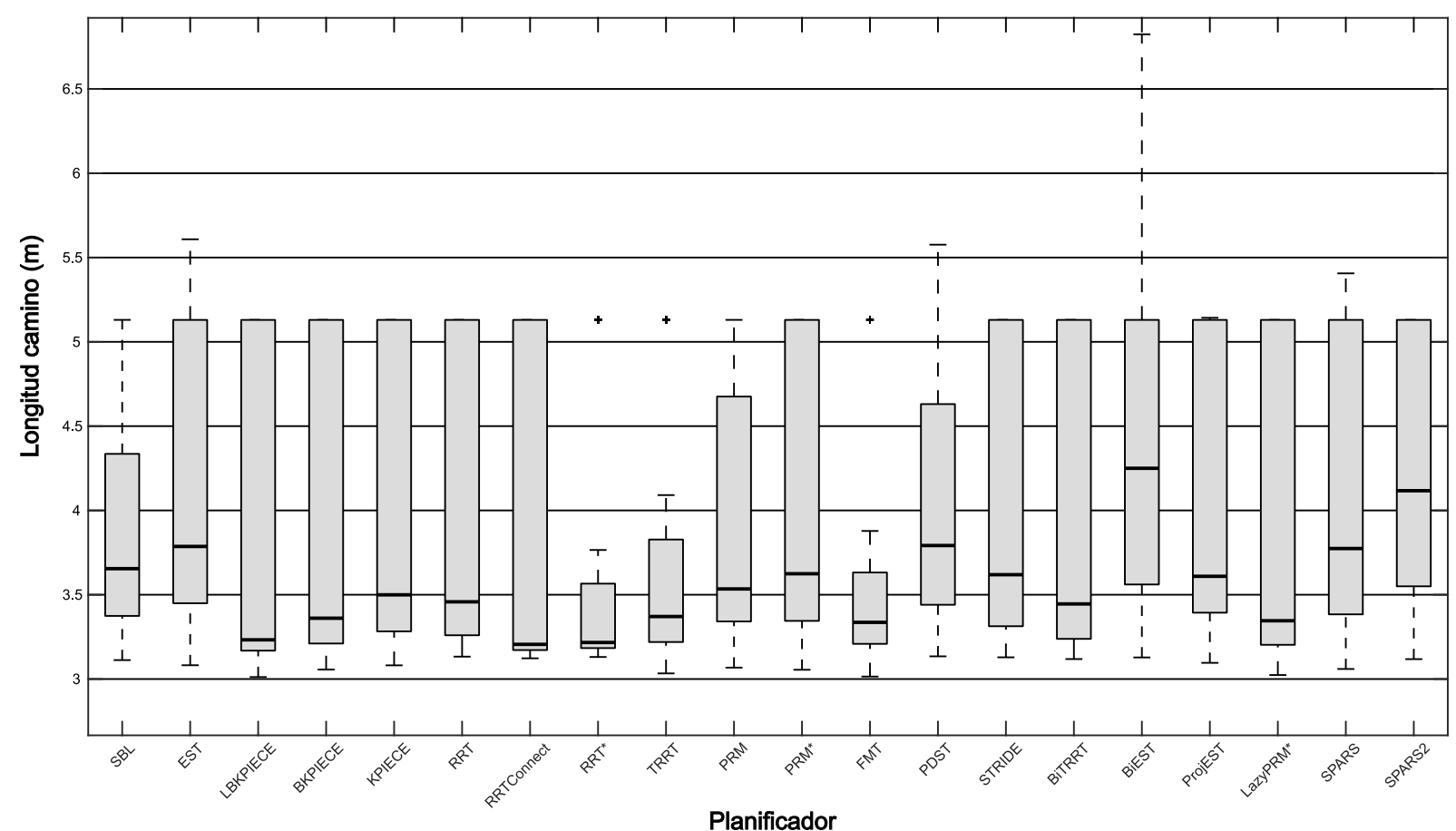

Figura 4: Longitud de trayectorias planificadas para tarea por encima de la cabeza

medio de 0.0689 segundos. Los planificadores SBL, EST, RRTConnect, STRIDE, BiTRRT, BiEST y ProjEST han sido capaces de encontrar un camino válido en un tiempo medio inferior a 0.1 segundos. Los planificadores más lentos han sido el RRT*, PRM, PRM*, LazyPRM*, SPARS y SPARS2, con un tiempo medio entorno a los 5 segundos. En cuanto a longitud de las trayectorias planificadas, la menor mediana se obtiene con el planificador RRTConnect $(3.2057 \mathrm{~m})$. No obstante, el menor rango intercuartílico se obtiene mediante el planificador RRT* (0.3823).

Para la tarea frontal, todos los planificadores han sido capaces de encontrar un camino válido en el tiempo máximo establecido excepto RRT (89\%), RRT* (96\%), TRRT (95\%) y LazyPRM 


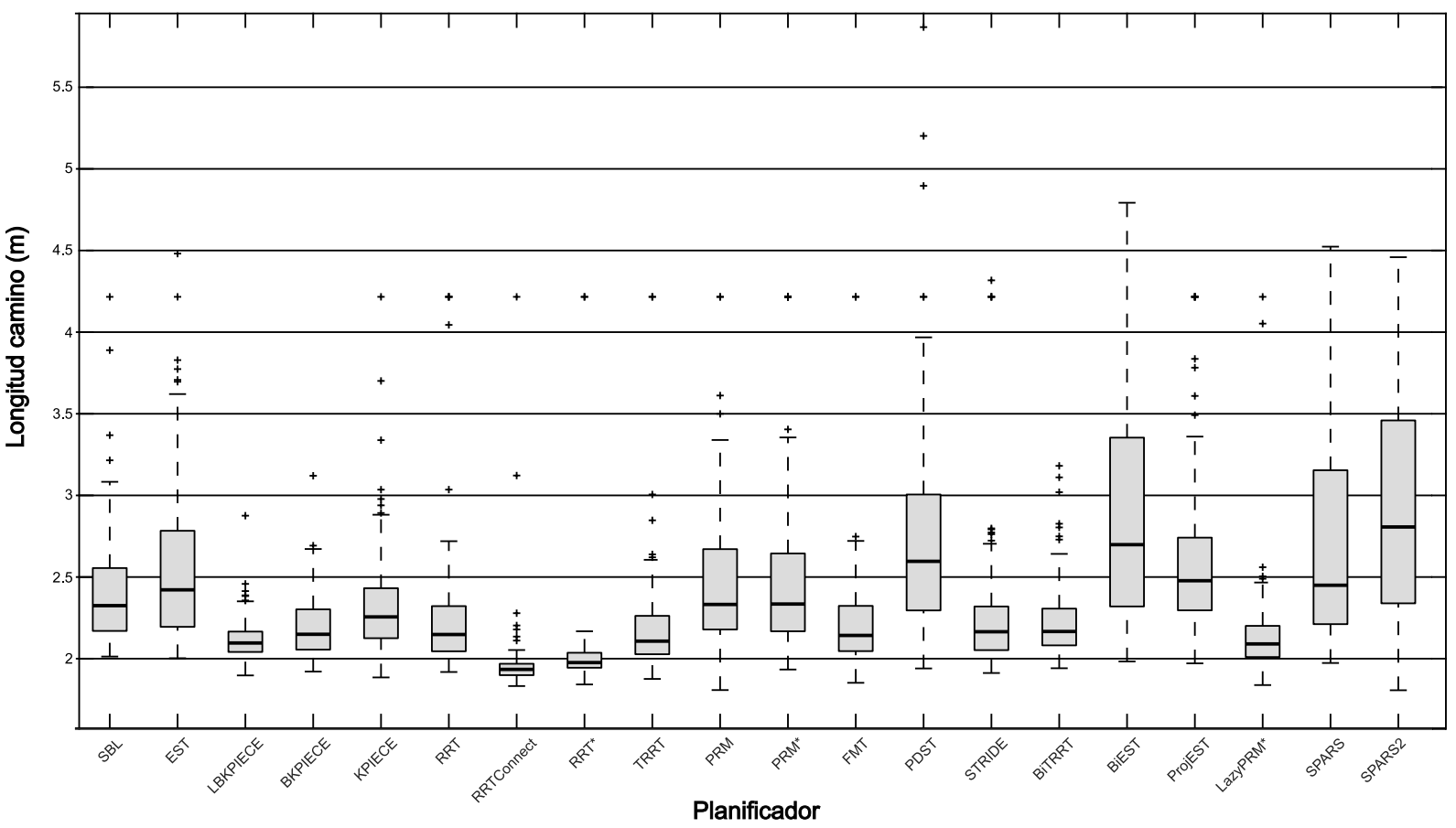

Figura 5: Longitud de trayectorias planificadas para tarea frontal

(0\%). El planificador más rápido ha sido STRIDE, con un tiempo de planificación medio de 0.0989 segundos, y el planificador KPIECE también ha sido capaz de obtener un tiempo de planificación medio inferior a los 0.1 segundos. Para esta tarea, el planificador RRTConnect ha generado trayectorias con la menor longitud mediana (1.9342 m), obteniendo también el menor rango intercuartílico (0.0697).

\section{DISCUSIÓN}

A la vista de los resultados, observamos que de forma general todos los planificadores obtienen un elevado porcentaje de éxito de planificación para ambas tareas. No obstante, empleando el planificador LazyPRM no se logró planificar ningún camino entre los puntos inicial y final para ninguna de las tareas presentadas.

Por otro lado, atendiendo a la longitud del camino planificado, el planificador RRTConnect ha obtenido la mediana más baja, tanto para la tarea sobre la cabeza como para la tarea frontal. Sin embargo, no ocurre lo mismo con el rango intercuartílico (IQR) obtenido para la longitud de las trayectorias. Si bien es cierto que para la tarea frontal el planificador RRTConnect obtiene el menor IQR, para la tarea sobre la cabeza se obtiene un IQR elevado, solo superado por el IQR del planificador LBKPIECE. Asimismo, cabe destacar que, para la tarea sobre la cabeza, ninguno de los planificadores empleados ha sido capaz de mantener longitudes inferiores a $5 \mathrm{~m}$ para todos los intentos de planificación, como se observa en la Figura 4. Para la tarea frontal, generalmente los planificadores no logran mantener todas las longitudes por debajo de $4 \mathrm{~m}$ (Figura 5). No obstante, el planificador LBKPIECE logra planificar todas las trayectorias con una longitud inferior a 3 m, y los planificadores BKPIECE y BiTRRT mantienen longitudes por debajo de $3.5 \mathrm{~m}$.

En cuanto a los tiempos de planificación, de forma general los planificadores single-query logran un tiempo de planificación medio entorno a los 0.1-0.2 segundos para ambas tareas. El planificador FMT se trata de una excepción, requiriendo un tiempo medio de 1.2154 y 1.3544 segundos. No obstante, los planificadores multi-query consumen el total del tiempo máximo establecido (5 segundos). A pesar de que para las tareas simuladas se asume un movimiento nulo por parte del operario, en otro tipo de tareas el continuo movimiento de los brazos del usuario provoca que el espacio libre varíe de forma continuada. Por ello, se ha decidido evaluar el problema de planificación como single-query, sin emplear el mapa de caminos generado y comprobando el efecto que tiene en el tiempo de planificación.

Por tanto, en base a las tareas simuladas, los planificadores LBKPIECE, BKPIECE, RRTConnect y BiTRRT muestran un buen rendimiento de acuerdo con el porcentaje de éxito, el tiempo de planificación requerido y las 
longitudes de las trayectorias planificadas.

\section{CONCLUSIÓN}

Se ha presentado un modelo simulado del sistema SecondArmS, implementado en ROS. Dicho modelo incluye dos manipuladores robóticos de 6 GDL, una plataforma elevadora y el propio usuario, modelando, además, el movimiento de los brazos humanos.

Mediante el modelo implementado se ha simulado 2 tareas de mantenimiento, y se ha evaluado el rendimiento de planificadores de trayectorias de la librería OMPL. A partir de los datos obtenidos, se puede concluir que los planificadores LBKPIECE, BKPIECE, RRTConnect y BiTRRT obtienen un mejor rendimiento en base al porcentaje de éxito, el tiempo de planificación y las longitudes de las trayectorias planificadas.

Sin embargo, cabe destacar que los resultados se han obtenido para posiciones fijas del brazo del operador humano. En un futuro se espera probar el sistema en un escenario real, obteniendo la posición en tiempo real de los brazos del usuario y empleando un prototipo del sistema SecondArmS. Asimismo, se analizará y evaluará en mayor profundidad los planificadores que muestran un mejor rendimiento.

\section{Agradecimientos}

Este estudio ha sido subvencionado por el Centro para el Desarrollo Tecnológico Industrial(CDTI) perteneciente al Ministerio de Ciencia e Innovación, a través del proyecto IDI-20190764 (Cofinanciado por el Fondo Europeo de Desarrollo Regional. FEDER, una manera de hacer Europa).

\section{Acrónimos}

BiEST Bidirectional Expansive Space Trees

BiTRRT Bidirectional Transition-based Rapidly-exploring Random Trees

BKPIECE Bidirectional Kinematic Planning by Interior-Exterior Cell Exploration

EST Expansive Space Trees

FMT Fast Matching Tree

KPIECE Kinematic Planning by Interior-Exterior Cell Exploration

LBKPIECE Lazy Bidirectional Kinematic Planning by Interior-Exterior Cell Exploration

PDST Path-Directed Subdivision Trees
PRM Probabilistic Road Map

ProjEST Projection-based Expansive Space Trees

RRT Rapidly-exploring Random Trees

SBL Single-query Bidirectional Lazy collision checking planner

SPARS SPArse Roadmap Spanner

STRIDE Search Tree with Resolution Independent Density Estimation

TRRT Transition-based RRT

English summary

Path planners analysis to avoid human user in supernumerary systems

\begin{abstract}
To decrease musculoskeletal disorder in the workplace, a supernumerary robot can be used to assist the human operator during maintenance tasks. Moreover, the supernumerary device is intended to move cooperatively with the human user in an autonomous or semi-autonomous way. For this reason, a system able to avoid collision with the user is needed. In this work, path planners are analyzed to avoid human arms during maintenance tasks with a simulated model.
\end{abstract}

Keywords: Supernumerary robot, path planners, ROS.

\section{Referencias}

[1] AGENCIA EUROPEA PARA AL SEGURIDAD Y LA SALUDA EN EL TRABAJO. Trastornos musculoesqueléticos. [Consulta de abril 2013].

[2] Bertomeu-Motos, A., Blanco, A., Badesa, F. J., Barios, J. A., Zollo, L., \& Garcia-Aracil, N. (2018). Human arm joints reconstruction algorithm in rehabilitation therapies assisted by end-effector robotic devices. Journal of neuroengineering and rehabilitation, 15(1), $1-11$. 
[3] Chitta, S., Sucan, I., \& Cousins, S. (2012). Moveit![ros topics]. IEEE Robotics \& Automation Magazine, 19(1), 18-19.

[4] INSTITUTO NACIONAL DE SEGURIDAD E HIGIENE EN EL TRABAJO. Riesgos de trastornos musculoesqueléticos en la población laboral española (2014).

[5] Norkin, C. C., \& White, D. J. (2016). Measurement of joint motion: a guide to goniometry. FA Davis.

[6] Quigley, M., Conley, K., Gerkey, B., Faust, J., Foote, T., Leibs, J., ... \& Ng, A. Y. (2009, May). ROS: an open-source Robot Operating
System. In ICRA workshop on open source software (Vol. 3, No. 3.2, p. 5).

[7] Sucan, I. A., Moll, M., \& Kavraki, L. E. (2012). The open motion planning library. IEEE Robotics \& Automation Magazine, $19(4), 72-82$.

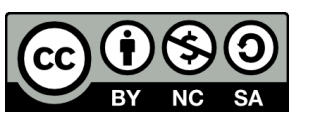

C) 2021 by the authors. Submitted for possible open access publication under the terms and conditions of the Creative Commons Attribution CC BY-NC-SA 4.0 license (https://creativecommons.org/licenses/by-nc-sa/4.0/deed.es). 